\title{
Optimal Prevention for Multiple Risks
}

\author{
Christophe Courbage \\ Geneva School of Business Administration \\ University of Applied Sciences Western Swizterland (HES-SO) \\ Henri Loubergé \\ University of Geneva \\ Geneva School of Economics and Management, Swiss Finance Institute \\ Richard Peter \\ Department of Finance \\ Henry B. Tippie College of Business, University of Iowa
}

\begin{abstract}
This paper analyzes optimal prevention in a situation of multiple, possibly correlated risks. We focus on probability reduction (self-protection) so that correlation becomes endogenous. If prevention concerns only one risk, introducing a second exogenous risk increases the level of prevention expenditures, even if correlation is negative. If prevention expenditures may be invested for both risks, a substitution effect arises. Under non-increasing returns on self-protection, we find that increased dependence increases aggregate prevention expenditures, but not necessarily prevention expenditures for each risk due to differences in prevention efficiency. Similar results are found when considering changes in the severity of losses. Consequently, the comparative statics emphasize global effects versus allocation effects. Our results have strong policy implications, considering the numerous mandatory safety measures introduced by governments over the past years.
\end{abstract}

Keywords: prevention - correlation - multiple risks

JEL-Classification: D81 - D91 


\section{Introduction}

Individuals and firms face multiple risks. Some of them are independent but others are dependent. For example, the risk of damage to the roof is often associated to the risk of flooding; the risk of releasing hazardous waste in the environment may be associated to the risk of suffering virus attacks on the information system; the risk of workers' injuries is known to be negatively correlated with the credit risk of consumers due to their common link to the business cycle. Some of the risks may be avoided by taking radical measures, for example giving up the pleasure of skiing to avoid the risk of a ski accident, or giving up the development of a new product to avoid potential product liability suits. When the risks cannot, or should not, be avoided they may be managed by prevention and loss reduction measures or transferred to an insurer. The paper focuses on prevention as a risk management tool.

Prevention is an ex-ante activity that reduces the probability of a loss. ${ }^{1}$ The economic analysis of prevention started with the seminal work of Ehrlich and Becker (1972) and has led since then to a flourishing literature (Courbage, Rey, and Treich, 2013). But, the canonical model, developed along several dimensions, assumes that the decision-maker (DM) faces only one risk to be mitigated using prevention measures, such as investing in fire-proof materials to reduce the probability of fire, or investing in locks and alarms to reduce the probability of burglary. Given that firms and individuals face multiple risks, the question we ask in this paper is how the decision to prevent one risk interacts with the decision to prevent other risks. More particularly, we wonder how the characteristics of the multiple risks and the efficiency of alternative prevention instruments influence the decision to invest in a specific portfolio of prevention measures. For instance, if we consider two risks and if the dependence between the two risks increases, will the DM increase his total investment and the level of both prevention activities? How will he alter the composition of his portfolio of prevention measures? Our analysis can also help to predict the effect of imposing mandatory prevention expenditures for one risk on the decision to prevent other risks.

While the literature on economic decisions in a multiple risk setting is quite abundant, only a few papers have recently addressed the study of self-protection in the presence of other risks. ${ }^{2}$ These papers look at either the relation between self-protection and risk aversion in the presence of an independent zero-mean background risk (Dachraoui, Dionne,

$1 \quad$ Following Ehrlich and Becker's (1972) terminology, this activity is also referred to as self-protection. Throughout the paper we use both terms synonymously.

2 Note, however, the contributions of Briys, Schlesinger, and v. d. Schulenburg (1991) and Schlesinger (1992) considering the reliability of prevention expenses. There is only one risk in the endowment, but the possible failure of prevention measures introduces an additional (multiplicative) risk. 
Eeckhoudt, and Godfroid, 2004), or the impact of an independent zero-mean background risk on self-protection activities in a one-period model (Lee, 2012) or a two-period model (Eeckhoudt, Huang, and Tzeng, 2012; Courbage and Rey, 2012; Wang and Li, 2015). Our paper differs markedly from the above literature in three ways. First, we allow for non-zero correlation between the different sources of risk. This is motivated, among other things, by recent empirical evidence (Sun and Frees, 2013). Second, we also consider the decision to prevent both risks simultaneously, and not merely the influence of the second risk on optimal prevention for the first risk. Third, we derive policy implications from our analysis by investigating the impact of exogenous changes in the level of prevention for one risk on the optimal self-protection investment for the other risk. This allows us to assess the side effects of the numerous mandatory safety measures introduced by governments and international organizations over the past years. ${ }^{3}$ We find that raising exogenously the level of protection for one risk induces a negative impact on the prevention expenditures for another unregulated risk.

To carry out our analysis, we draw on recent works that model prevention activities in a two-period expected utility framework. The idea behind this approach stems from the fact that the decision to engage in prevention activities precedes its effect on the risk, whereas the one-period model implicitly assumes that the decision to engage in prevention and its effect on the risk are simultaneous. Such a way to model prevention was introduced by Menegatti (2009) who investigates the role of prudence in the decision to develop prevention, and its popularity has since then steadily increased. It has produced new results on risk aversion and prevention (Menegatti, 2012), on the relationship between saving and prevention (Menegatti and Rebessi, 2011), it has been used to study the effect of the introduction of an independent zero-mean background risk on the decision to prevent a first risk (Eeckhoudt, Huang, and Tzeng, 2012; Courbage and Rey, 2012; Wang

3 For instance, it is now mandatory in most developed countries to install fire detectors (smoke alarms) at home. In the domain of vehicle safety regulation, the United Nations Economic Commission for Europe (UNECE) has been particularly active with recommendations ranging from safety belts to door locks and head restraints, among others. The European Commission (EC) has enacted a General Product Safety Directive (GPSD) requiring Member States to enforce among producers and distributors the obligation to place standardized products on the market and to inform consumers of the risks associated with the products they supply (see http://ec.europa.eu/consumers/archive/ safety/prod_legis/index_en.htm). Specific legislation exists for chemicals, toys, personal protective equipment, cosmetics, pharmaceuticals, machinery, recreational crafts, and lifts. Recently, new European standards have been announced to improve the safety of window blinds, following reports of fatal accidents involving young children strangulated by loosely hanging cords (see https://www.cen.eu/news/brief-news/pages/News-2014-005.aspx). These regulations aim at reducing the probability of costly accidents, but they impose higher costs for producers, distributors, and consumers. 
and Li, 2015), or in the context of preventive care (Menegatti, 2014). ${ }^{4}$ As we will point out later this idea of modeling optimal prevention intertemporally is isomorphic to an atemporal model in which the cost of prevention are separable. Our analysis illustrates that the main advantage of this formulation is that the marginal utility cost of preventive activity remains fixed as we vary parameters on the benefit side, which increases the tractability of our model.

A consequence is that our approach is quite different from the literature on optimal insurance purchasing under multiple correlated risks (Doherty and Schlesinger, 1983b; vd Schulenburg, 1986; Briys, Kahane, and Kroll, 1988). This also helps to explain why some of our results are very different from prior findings. In the insurance case, the main tool has been the canonical monoperiodic model of insurance demand as introduced by Mossin (1968) and Smith (1968) and extended in various directions. Here, both the marginal utility cost and the marginal utility benefit are affected by changes in exogenous parameters.

Our analysis proceeds along the following lines. First, we look at how the characteristics of one risk affect the decision to lower the probability of loss of the other risk. We show that introducing a second possibly correlated risk increases prevention for the endogenous risk, independently of the sign of correlation. We also show that if the overall risk faced by the DM is higher, through either a higher loss or a higher probability for the second risk, or a higher interdependence between the two risks, then the DM carries out more prevention with respect to the first risk. Secondly, when we consider the decision to prevent both risks simultaneously, we are able to define conditions under which the level of prevention directed at one risk is higher than the level of prevention directed at the other risk. Thirdly, we show that a substitution effect arises between both prevention measures, with important implications for regulation in the field of risk management. Our results do not replicate the results obtained in the literature on compulsory insurance in a multiple risk setting (vd Schulenburg, 1986) where the sign of correlation - positive or negative - plays a major role. This is due to reasons indicated above and will be made clearer in the course of the analysis. Fourthly, we consider the effect of interdependence on optimal expenditures on self-protection. It turns out that a higher dependence increases overall prevention expenditure, as expected, but the impact is not the same on both prevention instruments. It may happen that one kind of expense increases and the other is reduced. Similarly, an increase in the size of a loss for one risk increases overall prevention expenditure, but does not necessarily increase prevention expenses for the individual prevention instruments. To

4 Note, however, that two-period prevention models might collapse to their monoperiodic counterparts when saving is endogenized (Hofmann and Peter, 2015b). As we focus on prevention, we do not include saving in the analysis. 
the best of our knowledge, these effects have not been explicitly addressed in the existing literature so far.

The paper is organized as follows. In the next section, we introduce the general model. In section 3, we consider only one endogeneous risk and investigate how the decision to prevent this risk is influenced by the properties of the second risk. In section 4, we consider both risks to be endogeneous and compare the optimal level of prevention for each of them. Then we develop some comparative statics to address the interdependency between the two preventive activities. A short conclusion is provided in the last section.

\section{The General Model}

Let us consider a decision-maker confronted with two risks. The first risk shall be given as $\tilde{\epsilon}$ with outcomes $-l$ and 0 , i.e., the individual might suffer a loss of $l>0$. The probability of loss is given by $p \in(0,1)$. The second risk is symmetric in structure and is given by $\tilde{\zeta}$ with outcomes $-g$ and 0 , i.e., the individual might suffer a loss of $g>0$. The loss probability shall be denoted as $q \in(0,1)$ for the risk $\tilde{\zeta}$.

Regarding the joint distribution we allow for correlation between the two risks meaning that the distribution of $(\tilde{\epsilon}, \tilde{\zeta})$ need not necessarily be given by the product distribution of the two single risks. Consistent with Sun and Frees (2013), who find evidence of positive correlation between the relative frequency of homeowners and auto insurance claims, we do not want to limit ourselves to independent risks as most prior literature. To parametrize this situation of stochastic dependence, we introduce the parameter $k$ that describes to what extent the probability of occurrence of both losses deviates from the product of the individual loss probabilities. We call $k$ a measure of (inter)dependence and assume it to be exogenous to the analysis. ${ }^{5}$ With this convention, four states of nature are possible with their respective probabilities:

- Both losses of $l$ and $g$ with probability $k p q$,

- only the loss of $l$ with probability $p(1-k q)$,

- only the loss of $g$ with probability $q(1-k p)$,

- and no loss at all with probability $1-p-q+k p q$.

5 Naturally, when considering self-protection, correlation can no longer be exogenous. Inspired by similar treatments of the standard portfolio problem (see Gollier, 2001, chapter 4), we introduce an exogenous parameter to facilitate comparative statics analysis. Assuming $k$ to remain constant when $p$ (or $q$ ) changes with self-protection expenses amounts to assuming that the conditional probability of the loss $l$ ( or $g$ ) given that the loss $g$ (or $l$ ) has occurred changes in the same proportion as the unconditional probability. 
Doherty and Schlesinger (1983a) use a related framework to analyze the impact of correlated uninsurable background risks on the demand for insurance against insurable foreground risks. Rey (2003) extends their approach to the study of a nonpecuniary correlated background risk and Courbage and Rey (2007) use it to study the implications of correlation between a financial and a nonpecuniary risk on precautionary saving. One property of this formulation is that the marginal distributions for risk $\tilde{\epsilon}$ and $\tilde{\zeta}$ collapse to the wellunderstood distributions with two states of the world.

Unlike Eeckhoudt, Huang, and Tzeng (2012) who study prevention decisions in the presence of an independent second risk with a zero mean, our second risk has a negative mean because no outcome increases final wealth, and it is allowed to be correlated with the first risk. This is apparent when calculating the correlation coefficient between the two risks:

$$
\tau(\tilde{\epsilon}, \tilde{\zeta})=(k-1) \sqrt{\frac{p}{1-p} \cdot \frac{q}{1-q}} .
$$

Hence, a parameter of $k=1$ represents uncorrelated risks, $k>1$ positively correlated risks, and $k<1$ negatively correlated risks. ${ }^{6}$ Note that the strength of correlation is monotonically linked to the size of $k$ for given loss probabilities $p$ and $q$. Exploiting the fact that the probabilities of each event are between zero and one, we can determine thresholds for $k$. It is easy to see that $k$ must be in the following interval:

$$
\left[\max \left(0, \frac{p+q-1}{p q}\right), \min \left(\frac{1}{p}, \frac{1}{q}\right)\right] .
$$

In this sense, the plausible range for values of $k$ depends on both individual loss probabilities but it always contains $1 .^{7}$ Note that the case of $k=0=(p+q-1) / p q$ resembles perfect negative correlation $(\tau=-1)$ whereas if $k=1 / p=1 / q$ we obtain perfect positive correlation $(\tau=1)$. These are rather technical cases. Furthermore, if $k=0$, the probability of both losses and of the second loss only do not depend on the probability of the first loss.

Regarding the time structure of our model we assume two points in time, $t_{1}$ and $t_{2}$. We make this assumption as we follow some recent works where prevention is modeled in

6 Examples are floods and damages from wind which are positively correlated risks, or damages to one's home and vehicle accidents $(k>1)$. In a firm, credit risk and workers' injuries are negatively correlated risks, due to their dependence on the business cycle $(k<1)$.

7 It is hard to characterize it more explicitly because as soon as prevention is introduced, the parameter $k$ influences prevention expenditures which determine loss probabilities which determine the admissible $k$ values. This is related to the endogeneity of correlation in prevention problems. In the majority of applications the loss probabilities can safely be assumed to be below 0.5 so that the interval ranges from 0 to at least 2. 
a two-period setting (see Menegatti, 2009). ${ }^{8}$ We assume utility to be separable across time with first-period preferences given by the vNM utility function $u$, and second-period preferences given by the vNM utility function $v$. Utility is increasing in wealth in each period $\left(u^{\prime}()>\right.$.0 and $\left.v^{\prime}()>0.\right)$ and the individual is risk-averse in both periods $\left(u^{\prime \prime}()<0\right.$. and $\left.v^{\prime \prime}()<0.\right)$. Given these assumptions, the individual's expected utility reads as:

$$
u(w)+\mathbb{E} v\left(w_{2}+\tilde{\epsilon}+\tilde{\zeta}\right)
$$

To compress notation we define $w_{N N}:=w_{2}, w_{L N}:=w_{2}-l, w_{N L}:=w_{2}-g$, and $w_{L L}:=w_{2}-l-g$, where subscript $N$ denotes "no-loss" and subscript $L$ denotes "loss". The first letter of the subscript refers to the first risk $(\tilde{\epsilon})$, the second letter of the subscript refers to the second risk $(\tilde{\zeta})$. With this notation, expected utility becomes

$$
\begin{aligned}
u(w) & +(1-p-q+k p q) v\left(w_{N N}\right)+p(1-k q) v\left(w_{L N}\right) \\
& +q(1-k p) v\left(w_{N L}\right)+k p q v\left(w_{L L}\right)
\end{aligned}
$$

Furthermore, we use the conventions $\alpha:=v\left(w_{N N}\right)-v\left(w_{L N}\right), \beta:=v\left(w_{N L}\right)-v\left(w_{L L}\right)$, $\gamma:=v\left(w_{N N}\right)-v\left(w_{N L}\right)$, and $\delta:=v\left(w_{L N}\right)-v\left(w_{L L}\right)$. Note that $\alpha-\beta=\gamma-\delta<0$ due to risk aversion.

\section{One Endogenous Risk}

In this section, we study how the characteristics of one risk affect the decision to lower the probability of loss from another risk. We look at a situation where one of the two risks is endogenous, as the individual can invest in self-protection. Prevention expenditures of $x$ at $t_{1}$ lead to a reduction of the loss probability to $p(x)$ in the next period $t_{2}$. The individual's maximization problem is therefore given as

$$
\max _{x}\left\{\begin{aligned}
u(w-x)+ & (1-p(x)-q+k p(x) q) v\left(w_{N N}\right)+p(x)(1-k q) v\left(w_{L N}\right) \\
+ & q(1-k p(x)) v\left(w_{N L}\right)+k p(x) q v\left(w_{L L}\right)
\end{aligned}\right\}
$$

with associated first-order condition

$$
\begin{aligned}
T_{x} & =-u^{\prime}\left(w_{1}\right)-p^{\prime}(1-k q)\left(v\left(w_{N N}\right)-v\left(w_{L N}\right)\right)-k p^{\prime} q\left(v\left(w_{N L}\right)-v\left(w_{L L}\right)\right) \\
& =-u^{\prime}\left(w_{1}\right)-p^{\prime}(1-k q) \alpha-k p^{\prime} q \beta=0 .
\end{aligned}
$$

8 The important assumption is that we are able to separate the marginal cost of prevention from the marginal benefit. Another modeling approach to achieve this would be a monoperiodic set-up with a non-monetary cost of effort as indicated in the introduction. 
$T$ denotes the objective function and subscript $x$ indicates the respective derivative. $w_{1}$ is shorthand for the (endogenous) wealth level at $t_{1}$, i.e., $w_{1}=w-x$, and the second-order condition holds under risk aversion and standard assumptions on prevention technology, i.e., $p^{\prime}<0, p^{\prime \prime}>0$. Let $x^{*}$ denote the solution to the first-order condition, i.e., the optimal level of self-protection expenditures. ${ }^{9}$

The first term of the FOC represents the marginal utility cost of prevention, i.e., the reduction in first-period utility due to an infinitesimally small increase in self-protection expenditures. The second and third terms represent the marginal utility benefit of prevention, i.e., the expected increase of second period utility due to a reduction in the loss probability $p$. More precisely, the second term is the marginal benefit of prevention conditional on the loss $g$ not occurring while the third term is the marginal benefit conditional on the loss $g$ occurring. Also note that we can rearrange the marginal utility benefit to $-p^{\prime} \alpha+k p^{\prime} q(\alpha-\beta)$ where the first term describes the marginal benefit of self-protection in the absence of the second risk and the second one how the presence of the second risk enters the trade-off.

A straightforward question is how the presence of the exogenous second risk influences self-protection expenditures on the endogenous first risk. Let us compare the optimal decision under the absence of the exogenous risk to the situation characterized above. If the second risk is not present, the DM chooses to maximize

$$
V(x)=u(w-x)+p(x) v\left(w_{2}-l\right)+(1-p(x)) v\left(w_{2}\right)
$$

with associated first-order condition

$$
V_{x}=-u^{\prime}(w-\bar{x})-p^{\prime}(\bar{x}) \alpha=0,
$$

where $\bar{x}$ represents optimal self-protection expenditures in the single-risk case. Note that the second-order condition holds generically in this case. Next, we evaluate the first-order expression in the two-risk situation at the optimal self-protection expenditures in the single-risk situation. This yields

$$
\begin{aligned}
T_{x}(\bar{x}) & =-u^{\prime}(w-\bar{x})-p^{\prime}(\bar{x})(1-k q) \alpha-p^{\prime}(\bar{x}) k q \beta \\
& =p^{\prime}(\bar{x}) \alpha-p^{\prime}(\bar{x}) \alpha+p^{\prime}(\bar{x}) k q(\alpha-\beta) \\
& =p^{\prime}(\bar{x}) k q(\alpha-\beta) .
\end{aligned}
$$

$9 \quad$ By defining $c(x)=u(w)-u(w-x)$ and rewriting $T(x)=u(w)+\mathbb{E} v\left(w_{2}+\tilde{\epsilon}\right)-c(x)$, we see that the two-period self-protection model is isomorphic to a model with an increasing and convex cost function that is separable. 
This is strictly positive if $k$ is positive and zero if $k$ is zero. Due to the second-order condition this implies that the introduction of the second risk cannot reduce self-protection expenditures on the first risk and increases them in all cases where $k$ is positive. This is summarized in the following proposition.

Proposition 1. Optimal self-protection expenditures for risk $\tilde{\epsilon}$ increase when the exogenous risk $\tilde{\zeta}$ is introduced for any $k>0$. They remain constant if $k=0$.

It is not surprising that the dependence parameter $k$, positively linked to the correlation coefficient for given $p$ and $q$, has a positive influence on the difference between $x^{*}$ and $\bar{x}$. If $k$ increases, $T_{x}$ increases and so do self-protection expenses (see Proposition 2 below). What seems more surprising is to observe that $x^{*}>\bar{x}$, for any non-zero $k$, independently of the sign of correlation. It is well-known in the financial and insurance literature on multiple risks that a negative correlation coefficient has a mitigating impact on the overall risk faced by the DM. Schlesinger and Doherty (1985) note that the willingness to insure an insurable risk is reduced if a second negatively correlated risk introduces a strong diversification effect in the final wealth. In the limit, the demand for insurance is zero if the second risk offsets completely the insurable risk so that final wealth is non-random. This is a case of "homemade hedging" (see vd Schulenburg, 1986, Proposition 2). ${ }^{10}$ Our two-period model of self-protection is very different in that only the marginal benefit is affected by the introduction of the second risk, which explains the different result.

We can rewrite second-period expected utility in the presence of both risks in the following vein:

$$
(1-p(x)) v\left(w_{N N}\right)+p(x) v\left(w_{L N}\right)-q[\gamma+k p(x)(\delta-\gamma)] .
$$

The first part is expected utility when only the first risk is present. From this, a utility loss is subtracted due to the introduction of the second risk. Clearly, a risk-averse DM would be better off if the second risk was absent. Now what is the effect of dependence? In the case where $k=0$, correlation embraces its lowest value and the utility loss is a deadweight loss because it is not affected by changes in self-protection. Consequently, there will be no behavioral change even if the DM is worse off by the introduction of the second risk. As soon as $k$ is positive, there is a link between the marginal distribution of the first risk and the overall distribution. We can also see this in the utility loss: When self-protection increases, the probability of the first loss decreases so also the probability of both losses decreases. Similarly, when self-protection increases, the probability of not suffering the first loss increases and so does the probability of suffering only the second loss. From risk aversion we know that the reduction in utility from suffering a loss is

10 In this case, a positive demand for insurance would reduce the compensating effect, with no impact on expected wealth if the insurance premium is fair. This would be unfavorable for the DM. 
smaller when rich than when poor, i.e., when the other loss has not happened than when it has happened. Technically, $\delta>\gamma$ so that the net effect of self-protection on the utility loss is positive when $k>0$. So besides the effect from the single-risk case there is an additional incentive to invest in prevention because the utility loss from the second risk can be mitigated "en passant".

Our result also contrasts the finding in Eeckhoudt, Huang, and Tzeng (2012) that the introduction of an independent zero-mean background risk in the second period increases optimal prevention if and only if the DM is prudent. In our case the introduction of the second risk increases expenditures on self-protection (whenever $k>0$ ) by risk aversion alone, i.e., prudence is not required. The reason is that the second risk here is a loss risk so that it can only deteriorate (or leave unchanged) the outcomes from the first risk. By risk aversion it follows that the utility loss from suffering the first loss is larger when the second loss has occurred than when it has not, technically $\beta>\alpha$. As a result the first-order expression as a function of $x$ in the presence of the second risk is weakly above the first-order expression in its absence, and strictly for $k>0$. As a result optimal self-protection expenditures should increase indendent of the sign of the third utility derivative. $^{11}$

We make two observations before we proceed. Let us first note that introducing a correlated second risk induces regression towards no correlation resulting from the DM's behavioral reaction. ${ }^{12}$ Furthermore, Proposition 1 also holds for actuarially fair prevention for which the expenditures coincide with the expected monetary benefit ${ }^{13}$ so that expected intertemporal consumption is constant.

As a next step we study how properties of the risk $\tilde{\zeta}$ and specifically the extent of interdependence affect the selected level of the preventive investment. In this sense, we relate the riskiness of the second risk to the amount invested in self-protection for the first risk. The results are summarized in the following proposition.

11 A consequence of the fact that the second risk is a loss risk and has only non-positive outcomes is that its mean is negative unlike the second risk in Eeckhoudt, Huang, and Tzeng (2012). However, with risk aversion only this property alone is not sufficient for an increase in self-protection expenditures as a negative mean does not preclude strictly positive outcomes which would upset our reasoning. So the important property is really that the second risk is a loss risk.

12 When a second risk is introduced, self-protection expenditures will be adjusted and this in turn affects correlation. Technically, the correlation coefficient is increasing (decreasing) in the probability $p$ of loss $l$ if and only if $k>1(k<1)$. For this reason, introducing a positively correlated second risk leads to a fall in $p$ and a decrease in correlation, whereas introducing a negatively correlated second risk leads also to a fall in $p$, but an increase in correlation.

13 In this case, the self-protection technology must be linear with $p^{\prime}(x)=-1 / l$. 
Proposition 2. For $k>0$, the DM exerts more prevention on risk $\tilde{\epsilon}$ with

i) a larger loss probability for risk $\tilde{\zeta}$,

ii) a more severe loss for risk $\tilde{\zeta}$,

iii) increased dependence between the two risks.

Proof. The second-order condition is satisfied, i.e., $T_{x x}<0$. Hence, when varying an exogenous parameter, we have to inspect the sign of the derivative of the first-order condition with respect to this parameter. This yields

$$
\begin{aligned}
T_{x q} & =k p^{\prime}(\alpha-\beta)>0, \\
T_{x g} & =k p^{\prime} q\left(v^{\prime}\left(w_{N L}\right)-v^{\prime}\left(w_{L L}\right)\right)>0, \\
T_{x k} & =p^{\prime} q(\alpha-\beta)>0 .
\end{aligned}
$$

To explain the intuition behind Proposition 2, first note that the marginal utility cost of prevention is not affected by the variations under consideration. Hence, we focus on the marginal benefit to rationalize the results. We draw on the following figure that represents the marginal utility benefit of the decision to engage in self-protection in our set-up. ${ }^{14}$

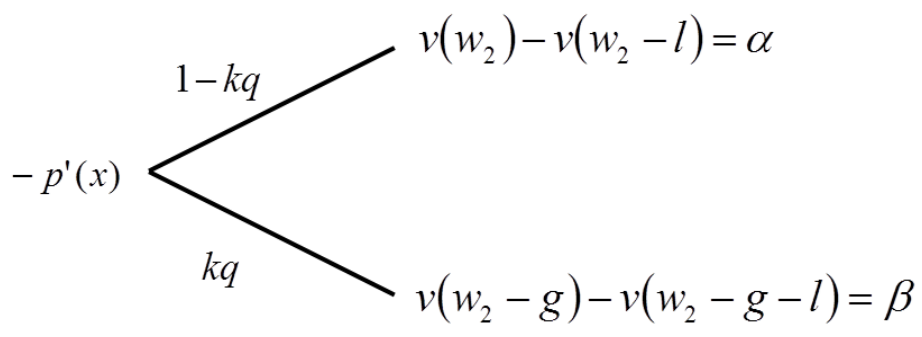

Figure 1 - Illustration of the marginal utility benefit

As said before, the marginal benefit has two components, one conditional on the loss $g$ not occurring (upper branch) and the other conditional on the loss $g$ occurring (lower branch). When $q$ increases, it decreases the weight of the marginal benefit of prevention when the loss $g$ has not occurred, and it increases the weight of the marginal benefit of prevention in the presence of the loss $g$. However, due to risk aversion, the utility difference between the states in which loss $g$ has not occurred $(\alpha)$ is smaller than the utility difference between the states in which loss $g$ has occurred $(\beta)$. This is the same type of argument as made before. Consequently, the net effect on the marginal utility benefit of prevention is positive implying that expenditures increase.

14 We thank Harris Schlesinger for suggesting this graphical approach. 
An increase in the loss size of the second risk only affects the marginal utility benefit conditional on this loss occurring. Due to diminishing marginal utility an increase in $g$ increases the pain from suffering the loss $l$, because the utility difference of a given income difference is larger at low wealth levels than at high wealth levels. Consequently, it is more beneficial to prevent the first loss knowing that the second loss has occurred. This explains the positive sign of $T_{x g}$.

Increasing interdependence shifts probability mass from the states of the world in which only one loss occurs to the states of the world where either both losses occur or neither does. $p^{\prime} q \alpha$ measures by how much the marginal benefit of preventing $l$ conditional on $g$ not occurring decreases due to an increase in dependence. The intuition is that with increased interdependence, knowing that $g$ has not occurred makes it less likely for $l$ to occur. Similarly, $-p^{\prime} q \beta$ measures the increase in the marginal benefit of preventing $l$ conditional on $g$ occurring due to increased dependence. Knowing that $g$ has occurred, increased interdependence makes it more likely that also $l$ will occur, so preventing this loss is more valuable. The overall effect is again positive due to the fact that risk aversion leads to a larger difference in utility conditional on $g$ having occurred than conditional on $g$ not having occurred. It becomes apparent that even with only one endogenous risk, the interdependence between the two risks influences the optimal course of action.

Proposition 2 says that if the overall risk faced by the individual is higher, through either a higher loss of risk $\tilde{\zeta}$, a higher loss probability of risk $\tilde{\zeta}$, or a higher dependence between the two risks, he carries out more prevention with respect to the risk $\tilde{\epsilon}$. In this sense, a correlated risk impacts optimal decisions even if it is completely exogenous, but we do not seem to observe any hedging effects because the sign of correlation is irrelevant for our findings. Some clarifications are in order. When investigating the value function of the DM's maximization problem, a direct application of the envelope theorem reveals that

$$
\frac{d T}{d k}\left(x^{*}\right)=T_{x}\left(x^{*}\right) \frac{\partial x}{\partial k}+T_{k}\left(x^{*}\right)=T_{k}\left(x^{*}\right)=p\left(x^{*}\right) q(\alpha-\beta),
$$

because self-protection is adjusted when dependence changes. Due to diminishing marginal utility we know that $\alpha<\beta$ so that, of course, the DM is worse off with stronger dependence. In this sense, there is a hedging effect when considering the individual's expected utility: Risk aversion implies that stronger negative correlation between the two risks is a good thing for the DM. Said differently, the DM's willingness to pay to decrease interdependence is strictly positive. Still, when it comes to his behavioral response, we see that in our two-period prevention model the sign of correlation does not play any specific role. 


\section{Both Risks Endogenous}

We now assume that both $p$ and $q$ may be reduced by expenditures $x$ and $y$, respectively, that are made in $t_{1}$. Moreover, we assume non-increasing returns on self-protection. We define the (technical) rate of return on self-protection expenditures $x$ by

$$
\rho(x):=\lim _{\Delta x \rightarrow 0}\left(\frac{p(x)-p(x+\Delta x)}{p(x)} \frac{1}{\Delta x}\right)=-\frac{p^{\prime}(x)}{p(x)},
$$

and analogously $\sigma(y)$ for loss prevention expenditures $y \cdot{ }^{15}$ Assuming non-increasing returns on self-protection means $\frac{d \rho(x)}{d x} \leq 0$, which is equivalent to $-\frac{p^{\prime}(x)}{p(x)} \leq-\frac{p^{\prime \prime}(x)}{p^{\prime}(x)}$. The assumption of non-increasing returns implies that prevention technologies are convex $\left(p^{\prime \prime}, q^{\prime \prime}>0\right)$, which is a standard assumption in the literature (Courbage, Rey, and Treich, 2013). Said differently, convexity is a necessary condition for having non-increasing returns on self-protection. In this sense, we have to strengthen the assumptions in our multiple risk setting slightly as compared to the analysis of prevention for single risks. Another comparison is the following: Whereas convexity assumes that the absolute decrease in loss probability does not increase, the assumption of non-increasing returns on self-protection corresponds to the fact that the percentage decrease in the loss probability does not increase. Rearranging the definition, we obtain for an infinitesimal increase in self-protection expenditures $\Delta x$ that $(p(x)-p(x+\Delta x)) / p(x)=\Delta x \cdot \rho(x)$; accordingly, as we spend more on self-protection the percentagewise reduction of the loss probability cannot become larger, see also Hofmann and Peter (2015a).

With this specification, the maximization problem is given by ${ }^{16}$

$$
\max _{x, y}\left\{\begin{array}{c}
u(w-x-y)+(1-p(x)-q(y)+k p(x) q(y)) v\left(w_{N N}\right) \\
+p(x)(1-k q(y)) v\left(w_{L N}\right)+q(y)(1-k p(x)) v\left(w_{N L}\right)+k p(x) q(y) v\left(w_{L L}\right)
\end{array}\right\}
$$

Let $T$ denote the objective function and let $w_{1}$ again be shorthand for the (endogenous) wealth level at $t_{1}$. Then, associated first-order conditions are given as

$$
\begin{aligned}
T_{x} & =-u^{\prime}\left(w_{1}\right)-p^{\prime}(1-k q)\left(v\left(w_{N N}\right)-v\left(w_{L N}\right)\right)-k p^{\prime} q\left(v\left(w_{N L}\right)-v\left(w_{L L}\right)\right) \\
& =-u^{\prime}\left(w_{1}\right)-p^{\prime}(1-k q) \alpha-k p^{\prime} q \beta=0, \\
T_{y} & =-u^{\prime}\left(w_{1}\right)-q^{\prime}(1-k p)\left(v\left(w_{N N}\right)-v\left(w_{N L}\right)\right)-k p q^{\prime}\left(v\left(w_{L N}\right)-v\left(w_{L L}\right)\right) \\
& =-u^{\prime}\left(w_{1}\right)-q^{\prime}(1-k p) \gamma-k p q^{\prime} \delta=0 .
\end{aligned}
$$

$15 \rho(x)$ measures by how much the loss probability decreases at the margin relative to the current probability of loss. Alternatively, it may be called the absolute decay rate of the loss probability.

16 As mentioned earlier, an alternative specification would be a model with separable cost of effort. If the disutility is an increasing and convex function of aggregate effort, all our results go through. 
The optimal expenditures on self-protection are denoted as $\left(x^{* *}, y^{* *}\right)$. Second-order conditions are assumed to hold for maximality. ${ }^{17}$ Note that the marginal utility cost of both self-protection instruments are equal. As a result, also the marginal benefits coincide at an optimum. Intuitively, a marginal dollar increases second-period expected utility by the same amount whichever technology it is invested in.

We first investigate whether Proposition 1 still holds when the second risk introduced is endogenous. By applying Proposition 1 to the risk $\tilde{\zeta}$ with probability $q\left(y^{* *}\right)$ and the given $k$, we confirm that also the introduction of an endogenous second risk raises optimal expenditures on the first risk for any positive $k$. Next we can study how expenditures for the risks $\tilde{\epsilon}$ and $\tilde{\zeta}$ compare to each other. Specifically, we investigate the conditions under which more money will be spent on preventing risk $\tilde{\epsilon}$ than risk $\tilde{\zeta}$. Intuitively, this depends on the rate of return of self-protection expenditures $x$ and $y$ and on the severity of the losses under consideration. The technical procedure for the comparison is provided in Gollier (2001), p. 151, and we will formulate it as a lemma.

Lemma 1. Let $f: \mathbb{R}^{2} \rightarrow \mathbb{R}$ be a concave function in the variables $(x, y)$ and $\left(x^{* *}, y^{* *}\right)$ be the local maximum implicitly defined via the first-order conditions. For a given $\underline{x} \in \mathbb{R}$ it holds that $x^{* *}>\underline{x}$ if and only if $f_{x}(\underline{x}, \hat{y})>0$ where $\hat{y}$ is the value that maximizes $f(\underline{x}, y)$.

Proof. See Hofmann and Peter (2015b).

We now apply Lemma 1 to the self-protection situation outlined above with two endogenous levels of expenditures. The result is summarized in the following proposition and we will develop some intuition afterwards.

Proposition 3. Prevention expenditures for risk $\tilde{\epsilon}$ will be larger than for risk $\tilde{\zeta}$ if and only if the marginal benefit of preventing $\tilde{\epsilon}$ is larger than the marginal benefit of preventing $\tilde{\zeta}$ when evaluated at $\left(y^{* *}, \hat{y}\right)$, with $\hat{y}$ solving $\max _{y} T\left(y^{* *}, y\right)$.

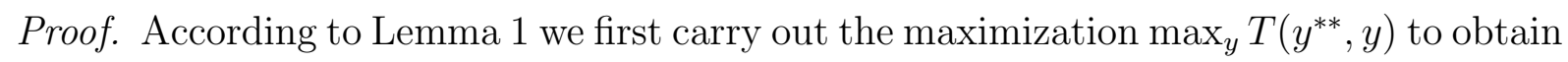
optimal expenditures $\hat{y}$ if $x$ is set to $y^{* *} . \hat{y}$ is defined via the first-order condition

$$
-u^{\prime}\left(w-y^{* *}-\hat{y}\right)-q^{\prime}(\hat{y})\left(1-k p\left(y^{* *}\right)\right) \gamma-k p\left(y^{* *}\right) q^{\prime}(\hat{y}) \delta=0 .
$$

17 Under the assumptions that the self-protection technology $x$ provides a return at least as large as selfprotection technology $y$, that returns on self-protection are non-increasing, and that $-q^{\prime \prime} / q^{\prime} \geq-p^{\prime} / p$, which we will introduce later to prove Proposition 6, one can show that the second-order conditions are satisfied, see the appendix. 
Now the first-order expression with respect to $x$ evaluated at $\left(y^{* *}, \hat{y}\right)$ is given by

$$
\begin{aligned}
T_{x}\left(y^{* *}, \hat{y}\right) & =-u^{\prime}\left(w-y^{* *}-\hat{y}\right)-p^{\prime}\left(y^{* *}\right)(1-k q(\hat{y})) \alpha-k p^{\prime}\left(y^{* *}\right) q(\hat{y}) \beta \\
& =q^{\prime}(\hat{y})\left(1-k p\left(y^{* *}\right)\right) \gamma+k p\left(y^{* *}\right) q^{\prime}(\hat{y}) \delta-p^{\prime}\left(y^{* *}\right)(1-k q(\hat{y})) \alpha-k p^{\prime}\left(y^{* *}\right) q(\hat{y}) \beta \\
& =M B_{x}\left(y^{* *}, \hat{y}\right)-M B_{y}\left(y^{* *}, \hat{y}\right),
\end{aligned}
$$

where $M B_{x}\left(M B_{y}\right)$ denotes the marginal benefit arising from prevention expenditures $x(y)$.

By inspecting the technical condition further one can isolate two effects which we summarize in the following corollary.

Corollary 1. The comparison of prevention expenditures for risk $\tilde{\epsilon}$ and for risk $\tilde{\zeta}$ involves two effects,

- a comparative efficiency effect,

- and a comparative loss size effect.

To see this rearrange $T_{x}\left(y^{* *}, \hat{y}\right)$ in the following way:

$$
(\alpha-\beta) k\left(p^{\prime}\left(y^{* *}\right) q(\hat{y})-p\left(y^{* *}\right) q^{\prime}(\hat{y})\right)+\left(q^{\prime}(\hat{y}) \gamma-p^{\prime}\left(y^{* *}\right) \alpha\right)
$$

The first summand describes a comparative efficiency effect. Under the assumption that $\rho\left(y^{* *}\right) \geq \sigma(\hat{y})$, it is non-negative indicating that more prevention should be carried out for risk $\tilde{\epsilon}$ than for risk $\tilde{\zeta}$. Furthermore, this effect is increasing in the interdependence between the two risks $(k)$ meaning that the more the two risks are correlated the more weight has to be attached to the comparison of the efficiency of the two technologies. Lastly, this effect is also related to the comparison of the loss in utility when going from one loss to two losses $(\alpha-\beta)$. The more painful it is to suffer the second loss the more relevant becomes this comparative efficiency effect.

However, it is not sufficient to have one technology more efficient than the other to arrive at an unambiguous result. The second effect relates the marginal benefit of prevention expenditures in the absence of the other loss. It is positive if and only if

$$
-p^{\prime}\left(y^{* *}\right)\left(v\left(w_{N N}\right)-v\left(w_{L N}\right)\right)>-q^{\prime}(\hat{y})\left(v\left(w_{N N}\right)-v\left(w_{N L}\right)\right)
$$

so that the relative loss sizes are decisive. In short, the overall sign of $T_{x}\left(y^{* *}, \hat{y}\right)$ is ambiguous since the second term might be negative even if $x$ provides more prevention efficiency than $y$. In the following, we illustrate two special cases in a corollary. Both are conditional on evaluation at $(x, y)=\left(y^{* *}, \hat{y}\right)$ as prescribed by Lemma 1 . 
Corollary 2. Assume equal loss sizes; with a lower loss probability, but higher marginal loss probability for risk $\tilde{\zeta}$ than risk $\tilde{\epsilon}$, expenditures to prevent $\tilde{\epsilon}$ are larger. Assume equal loss probabilities; then, higher prevention expenditures will be incurred for the risk with the larger loss size.

The intuition is fairly straightforward. In the first case, the second effect in Corollary 1 is nil because both losses are equally severe. Then, the technology with the lower probability of loss but the higher marginal probability of loss provides more prevention efficiency so that more money should be spent to prevent this risk. In the second case, prevention efficiency is identical for both technologies so that the comparative loss size effect is decisive. Finally we remark that little can be said about the relative strength of the two effects in the generic case.

\section{Comparative Statics}

Next we study how an optimal bundle of prevention expenditures reacts to changes in exogenous variables. In order to look at the interaction between the two decisions we first look at the reaction functions, i.e., we investigate how the optimal expenditures to protect against one risk react to a change in the expenditures to protect against the other risk and inversely. The joint optimum is obtained when both curves intersect. The following observation is of crucial importance and will be utilized in the rest of the paper.

Proposition 4. In equilibrium there is a substitution effect between preventive expenditures for risk $\tilde{\epsilon}$ and for risk $\tilde{\zeta}$.

Proof. Differentiating $T_{x}$ with respect to $y$ and $T_{y}$ with respect to $x$ gives:

$$
T_{x y}=T_{y x}=u^{\prime \prime}(w-x-y)+k p^{\prime} q^{\prime}(\alpha-\beta)<0
$$

for $u^{\prime \prime}<0$ and $v^{\prime \prime}<0$. This means that the optimal value of $x$ decreases following an exogenous increase of $y$ and conversely.

One direct consequence of the substitution effect is that in case of multiple equilibrium pairs that maximize the DM's utility we obtain that the equilibrium prevention expenditures for one risk are a decreasing function of the equilibrium prevention expenditures for the other risk. Loosely speaking we could say that the equilibrium pairs exhibit antimonotonicity. This result corresponds to Menegatti and Rebessi's (2011) for prevention and saving. Note that multiple equilibria are only possible if the second-order condition does not hold globally. The appendix provides sufficient conditions for obtaining a unique equillibrium.

The intuition behind the substitution effect between the two prevention measures is 
twofold. First, resources are scarce and with an exogenous increase of expenditures on one prevention measure first-period consumption is lower. This increases the marginal cost of prevention for the other measure. Second, the marginal benefit is affected. The marginal benefit conditional on the other loss not occurring is increased due to the fact that higher prevention expenditures to prevent the other loss make the state of the world in which it does not occur more likely. Similarly, the marginal benefit conditional on the other loss occurring is decreased because higher prevention expenditures to prevent the other loss make the state of the world in which it does occur less likely. The overall effect is negative, i.e., the second effect prevails, again due to diminishing marginal utility.

Note that this result is independent of the sign of correlation and has public policy implications. It implies that there are compensating reactions when the government imposes mandatory prevention expenditures on one kind of risk, for example fire, car accidents, or elevator accidents. ${ }^{18}$ Individuals and firms react by cutting self-protection expenditures on other risks. Therefore, the policymaker needs to take these reactions into account when changing safety requirements for specific risks, as unintentional increases in the exposure to other risks might decrease or even outweigh the gains from increased safety for the first risk. For reasons explained in the previous section, this substitution result is in contrast with comparable results in the literature on the demand for insurance with multiple risks. There, mandatory insurance requirements for one risk increase the insurance demand for the other risk if the two risks are negatively correlated (vd Schulenburg, 1986). We will repeatedly draw on the substitution effect for the remainder of the comparative statics analysis.

\subsection{Effect of Interdependence}

We start with dependence to analyze how the optimal values of both $x$ and $y$ vary when the extent of interdependence is increased. ${ }^{19}$ Intuitively one could think that when risks become more positively correlated, the expenditures to reduce the probability of each one increase. But as we know from Proposition 4, substitution between both measures of prevention might upset this effect. To smooth the exposition we make the assumption that without loss of generality expenditures $x$ provide a return on self-protection at least

18 An example is mandatory safety equipment that vehicle drivers in many countries have to carry with them. For more examples, see Footnote 3.

19 Note that

$$
\frac{d \tau}{d k}=\sqrt{\frac{p}{1-p} \cdot \frac{q}{1-q}}\left(1+\frac{k-1}{2}\left[\frac{1}{p(1-p)} \frac{\partial x}{\partial k}+\frac{1}{q(1-q)} \frac{\partial y}{\partial k}\right]\right),
$$

so that increased interdependence as measured by $k$ might lower or increase the correlation coefficient $\tau$ when behavioral adjustments are taken into account. At the no-correlation case, i.e., for $k=1$ the analogy is perfect meaning that marginal increases of $k$ are associated with marginal increases in the correlation coefficient and vice versa. 
as large as expenditures $y .{ }^{20}$

Totally differentiating the two first order conditions with respect to $k$ and applying the implicit function rule yields:

$$
\operatorname{sgn}\left(\frac{d x}{d k}\right)=\operatorname{sgn}\left(-T_{y y} T_{x k}+T_{x y} T_{y k}\right) .
$$

We drop the arguments of utility to compress notation and rearrange as follows:

$$
-T_{y y} T_{x k}+T_{x y} T_{y k}=(\alpha-\beta)\left[-u^{\prime \prime}\left(p^{\prime} q-p q^{\prime}\right)+(\alpha-\beta) k p p^{\prime}\left(\left(q^{\prime}\right)^{2}-q q^{\prime \prime}\right)+\gamma p^{\prime} q q^{\prime \prime}\right] .
$$

The sign of the first summand in squared brackets is determined by $\left(p^{\prime} q-p q^{\prime}\right)$ and depends on the relative efficiency of the two prevention opportunities. It is non-positive under the assumption that the return on self-protection for expenditures $x$ is at least as large as for expenditures $y$. The sign of the second summand in squared brackets depends on the expression $\left(q^{\prime}\right)^{2}-q q^{\prime \prime}$. It is non-positive whenever $-\frac{q^{\prime}}{q} \geq-\frac{q^{\prime \prime}}{q^{\prime}}$. This is equivalent to the assumption of non-increasing returns on self-protection as presented in section 4 . Note that, although we are interested in how $x$ is affected, we make the assumption that prevention technology $y$ satisfies the non-increasing returns property. Intuitively, the fact that $y$ loses efficiency when used more reinforces the use of $x$ and vice versa. Finally, the last term in squared brackets is unambiguously negative and measures the direct impact of increased interdependence on self-protection expenditures $x$. As a result the squared bracket is negative; multiplied by $(\alpha-\beta)$, which is itself negative, the overall sign is positive.

Hence, increased dependence between the two risks enhances prevention expenditures for the more efficient technology. From above we see that the sign of $d x / d k$ is driven by two competing effects. As demonstrated in Proposition 2, there is a direct effect that leads to more prevention with increased dependence, because the increase in marginal utility benefit of prevention on one risk conditional on the other risk occurring is larger than the decrease in marginal utility benefit of prevention on one risk conditional on the other risk not occurring. This is contained in $-T_{y y} T_{x k}$ which is unambiguously positive. However, an increase in prevention on one risk increases the marginal utility cost and lowers the marginal utility benefit for preventing the other risk, i.e., $T_{x y}<0$, so there

20 More precisely, as we study the impact of interdependence in this subsection, we take into account that each level of dependence $k$ implies optimal self-protection expenditures $x^{*}(k)$ and $y^{*}(k)$ according to the first-order conditions. Thus we can define the rate of return on self-protection as function of $k$, i.e., $\rho(k):=\rho\left(x^{*}(k)\right)$ and $\sigma(k):=\sigma\left(y^{*}(k)\right)$ and postulate that $\rho(k) \geq \sigma(k) \forall k$. This means that technology $x$ provides a return on self-protection which is at least as large as that of technology $y$ for all respective pairs of optimal self-protection expenditures. 
is a substitution effect, see Proposition 4. As demonstrated above the assumptions of $x$ providing a return on self-protection at least as large as $y$ and non-increasing returns to self-protection are sufficient to guarantee that for the more efficient technology the direct effect prevails.

Similarly, we can determine how prevention expenditures for $q$ react to changes in the interdependence measure $k$. This yields

$$
\operatorname{sgn}\left(\frac{d y}{d k}\right)=\operatorname{sgn}\left(T_{x y} T_{x k}-T_{x x} T_{y k}\right)
$$

with

$$
T_{x y} T_{x k}-T_{x x} T_{y k}=(\alpha-\beta)\left[u^{\prime \prime}\left(p^{\prime} q-p q^{\prime}\right)+(\alpha-\beta) k q q^{\prime}\left(\left(p^{\prime}\right)^{2}-p p^{\prime \prime}\right)+\alpha p p^{\prime \prime} q^{\prime}\right] .
$$

The analysis is as above. Note, however, that the first summand of the expression in squared brackets is non-positive as we assume $y$ to provide a return on self-protection not exceeding that of $x$. This explains why the effect of increased dependence on $y$ is ambiguous.

Lastly, we can determine how overall prevention expenditures react to changes in the level of interdependence. Let $D$ be the determinant of the Hessian; then, after some simplifications and using the definitions given above we arrive at

$$
\begin{aligned}
\frac{d(x+y)}{d k} & =\frac{d x}{d k}+\frac{d y}{d k} \\
& =-\frac{1}{D}(\alpha-\beta)^{2} k p p^{\prime} q q^{\prime}\left[\frac{\rho^{\prime}}{\rho}+\frac{\sigma^{\prime}}{\sigma}\right]-\frac{1}{D}(\alpha-\beta) p q\left[\alpha \sigma p^{\prime \prime}+\gamma \rho q^{\prime \prime}\right],
\end{aligned}
$$

which is unambiguously positive. Note that the comparative efficiency effects cancel out so only the effects due to non-increasing returns on self-protection and the direct effects remain. We summarize the analysis in the following proposition.

Proposition 5. Increasing dependence increases aggregate prevention expenditures under non-increasing returns on self-protection. It increases also expenditures on the more efficient technology, whereas the effect on the less efficient technology is ambiguous.

These results highlight the specificity of self-protection when considered in a multiple risks setting. The comparative efficiency of alternative prevention instruments plays a major role and may lead to observe a simultaneous increase in the use of one instrument and a decrease in the use of the other instrument although the two risks have become more interdependent. Furthermore, Proposition 5 illustrates that aggregate effects are more pronounced then allocation effects. 


\section{$5.2 \quad$ Impact of Loss Size}

We now study the question how changes in the severity of loss affect the optimal amount of self-protection expenditures carried out by the agent. One might intuitively think that an increase in the size of the loss increases prevention expenditures for this risk, but decreases expenditures for the other risk due to substitution between the two prevention opportunities. This assumption is, however, misleading due to the fact that increasing the loss size of one risk also exerts a positive effect on prevention expenditures for the other risk, as stressed in Proposition 2. In this subsection, we investigate the conditions to arrive at an unambiguous result.

Totally differentiating the first-order conditions with respect to $l$ and applying the implicit function theorem yields:

$$
\operatorname{sgn}\left(\frac{d x}{d l}\right)=\operatorname{sgn}\left(-T_{y y} T_{x l}+T_{x y} T_{y l}\right) .
$$

As already mentioned, the first term $\left(-T_{y y} T_{x l}\right)$ indicates a positive direct effect, whereas the second term $\left(T_{x y} T_{y l}\right)$ a negative substitution effect. To shed light on the net effect, we use the following rearrangement:

$$
\begin{aligned}
-T_{y y} T_{x l}+T_{x y} T_{y l}= & u^{\prime \prime}\left[k\left(v_{L L}^{\prime}-v_{L N}^{\prime}\right)\left(p^{\prime} q-p q^{\prime}\right)+p^{\prime} v_{L N}^{\prime}\right] \\
& +k^{2} p p^{\prime}(\alpha-\beta)\left(v_{L N}^{\prime}-v_{L L}^{\prime}\right)\left[\left(q^{\prime}\right)^{2}-q q^{\prime \prime}\right] \\
& +\left(k p^{\prime} q q^{\prime \prime} \gamma\left(v_{L N}^{\prime}-v_{L L}^{\prime}\right)+k p p^{\prime} q^{\prime \prime}(\alpha-\beta) v_{L N}^{\prime}-p^{\prime} q^{\prime \prime} \gamma v_{L N}^{\prime}\right) .
\end{aligned}
$$

The first term describes a comparative efficiency effect that favors the more efficient prevention technology. Under the assumption that $x$ provides a return on self-protection as least as large as $y$, this effect is positive indicating that expenditures on $x$ should be increased. The second term is again non-negative under the assumption of non-increasing returns on self-protection. As before, although we focus on the first risk here, this assumption has to be made about the technology addressing the second risk. Finally, the last term is unambiguously positive reflecting the initial intuition. Hence, we can conclude that an increase in the loss size $l$ unambiguously increases loss prevention expenditures on $x$ under the assumptions made. In this sense, if $x$ provides a return on self-protection at least as high as $y$ does, the direct positive effect dominates the negative substitution effect and therefore, if the loss associated with risk $\tilde{\epsilon}$ becomes more severe, this unambiguously leads to an increase of the self-protection investment $x$.

Next let us analyze how an increase in $l$ affects prevention expenditures on $y$. Proposition 2 gives rise to the conjecture that with a larger loss for risk $\tilde{\epsilon}$ also prevention expenditures regarding risk $\tilde{\zeta}$ should increase. However, due to the substitution effect between the two 
types of expenditures matters are more complex. The implicit function rule implies

$$
\operatorname{sgn}\left(\frac{d y}{d l}\right)=\operatorname{sgn}\left(T_{x y} T_{x l}-T_{x x} T_{y l}\right),
$$

Similar to above, $-T_{x x} T_{y l}$ is a positive direct effect whereas $T_{x y} T_{x l}$ represents a negative substitution effect. We can rearrange this as follows:

$$
\begin{aligned}
T_{x y} T_{x l}-T_{x x} T_{y l}= & u^{\prime \prime}\left[k\left(p^{\prime} q-p q^{\prime}\right)\left(v_{L N}^{\prime}-v_{L L}^{\prime}\right)-p^{\prime} v_{L N}^{\prime}\right] \\
& +k^{2} q q^{\prime}(\alpha-\beta)\left(v_{L N}^{\prime}-v_{L L}^{\prime}\right)\left[\left(p^{\prime}\right)^{2}-p p^{\prime \prime}\right] \\
& -k\left(p^{\prime}\right)^{2} q^{\prime}(\alpha-\beta) v_{L N}^{\prime}+k p p^{\prime \prime} q^{\prime}\left(v_{L N}^{\prime}-v_{L L}^{\prime}\right) \alpha .
\end{aligned}
$$

The first term describes a comparative efficiency effect again which favors the more efficient prevention technology. Under the assumption that $x$ provides a rate of return on self-protection at least as large as $y$, this summand is negative. The second term is about the non-increasing rate of return on self-protection for the technology to prevent the first risk. With our assumptions it is non-negative. The third term is negative, whereas the last term is positive. Hence, the overall sign is ambiguous.

Finally we can analyze how changes in the loss size $l$ influence overall prevention expenditures $x+y$. We obtain that

$$
\begin{aligned}
\frac{d(x+y)}{d l}= & \frac{d x}{d l}+\frac{d y}{d l} \\
= & -\frac{1}{D} k^{2}(\alpha-\beta)\left(v_{L N}^{\prime}-v_{L L}^{\prime}\right) p p^{\prime} q q^{\prime}\left[\frac{\rho^{\prime}}{\rho}+\frac{\sigma^{\prime}}{\sigma}\right] \\
& -\frac{1}{D} k p q\left(v_{L N}^{\prime}-v_{L L}^{\prime}\right)\left(\rho q^{\prime \prime} \gamma+\sigma p^{\prime \prime} \alpha\right) \\
& -\frac{1}{D} k p p^{\prime} q^{\prime}(\alpha-\beta) v_{L N}^{\prime}\left(-\frac{q^{\prime \prime}}{q^{\prime}}+\frac{p^{\prime}}{p}\right)-\frac{1}{D} p^{\prime} q^{\prime \prime} v_{L N}^{\prime} \gamma
\end{aligned}
$$

Note that the comparative efficiency effects cancel out again. The first summand here is non-negative due to the assumption of non-increasing returns to self-protection. The second is unambiguously positive because non-increasing returns to self-protection imply convexity as mentioned in section 4 . The third one is positive under the assumption that $-q^{\prime \prime} / q^{\prime} \geq-p^{\prime} / p$. The intuition behind this assumption is that $x$ provides more efficiency not only in a first-order but also in a second-order sense, see also Footnote 21 in the appendix. The fourth term is always positive again due to convexity of prevention technology. Hence, under the assumptions outlined above overall prevention expenditures rise when the severity of losses is increased. We summarize our results in the following proposition. 
Proposition 6. Assume a marginal increase in the size of the loss; if returns to selfprotection are non-increaseing and if the prevention instrument associated with the larger loss is the more efficient one, it experiences an increase in prevention expenditures. If in addition it is sufficiently more efficient, aggregate prevention expenditures increase.

Again, these results are original, compared to results obtained in previous literature on hedging/insuring against losses in a single-period context. In this literature, an increased loss size has an unambiguously positive effect on the demand for hedging. The specificity of prevention lies in the relative efficiencies of different prevention technologies to preserve final wealth. The consideration of relative efficiency is decisive in our set-up, because we can safely focus on the marginal utility benefit when conducting comparative statics analysis. As a result, increasing the loss size does not necessarily increase prevention expenditure to protect against that loss. In our model, increasing loss $g$ does not necessarily increase expenditure on $y$ if $x$ is more efficient than $y$. Similar to Proposition 5, we can see from Proposition 6 that aggregate effects are more pronounced than allocation effects.

\section{Conclusion}

This paper examines decision-makers' expenditures to mitigate risks in a situation of multiple risks. Specifically, we investigate self-protection investments in the sense of Ehrlich and Becker (1972), i.e., expenses to reduce the probability of loss. Following recent contributions in the literature (Menegatti, 2009; Eeckhoudt, Huang, and Tzeng, 2012; Courbage and Rey, 2012) we model prevention as investment preceding the reduction of the loss probability.

We first identify how characteristics of one risk affect the amount devoted to lowering the loss probability of another risk. We find that introducing a second correlated risk increases prevention for the endogenous risk, independently of the sign of correlation. We also find that increased riskiness in terms of the loss probability or the loss severity of one risk or the extent of interdependence between the two risks are associated with larger loss prevention investments for the other risk. We then proceed by analyzing a situation in which loss prevention can be carried out for both risks. By comparing the marginal utility benefit for both prevention opportunities at a specific level of investment we can decide for which risk the larger investment shall be incurred. This condition translates into two sub-conditions relating efficiency of prevention technology and the isolated benefit to one another.

We also find that a substitution effect between both measures of prevention arises. This has two reasons: First, by modeling prevention as investment preceding its benefit there is 
competition for resources between the two prevention opportunities. Second, the marginal benefit conditional on the other loss not occurring increases, whereas the marginal benefit conditional on the other loss occurring decreases, but the second effect prevails so that the overall marginal utility benefit decreases. This implies that exogenous increases of expenditures on one prevention measure due to regulation for example should be accompanied by endogenous decreases of expenditures on other prevention measures.

Finally, we conduct comparative statics analysis regarding our measure of interdependence and the size of potential losses. If dependence is increased, the marginal effect on the individual prevention measures can be decomposed into three components. There is a comparative efficiency component, an effect due to non-increasing returns on self-protection, and a positive direct effect. As the comparative efficiency effects exhibit opposite signs, overall prevention expenditures increase following an increase in interdependence. Furthermore, larger expenditures will be devoted to the more efficient technology, whereas the investment into the less efficient one might increase or decrease. When it comes to the size of the loss, we can again decompose the marginal effects into three components analogous to the study of dependence. The comparative efficiency effect favors the more efficient technology. If the prevention technology that is more efficient is associated with the increased loss, more resources will be incurred to prevent this risk. Moreover we identify suitable technical conditions that guarantee that overall expenditures increase. Again, the behavior of the less efficient technology is ambiguous.

Several extensions seem promising. A natural first thought would be to wonder whether and how the results obtained carry over to self-insurance or loss reduction. Following Ehrlich and Becker (1972) self-insurance refers to an investment that reduces the size of a given loss but leaves its probability of occurrence unaffected. It is well-known in the literature that self-insurance and self-protection may behave quite differently (Dionne and Eeckhoudt, 1985). Another avenue would be to investigate correlated risks that appear in different arguments of the utility function, i.e., a monetary risk flanked by a health risk. Tools of multivariate utility analysis (Rey, 2003; Lee, 2012) could then prove helpful to investigate the impact of correlation. It would also be interesting to analyze how comparative risk aversion impacts prevention expenses in a multiple risk setting by using results on risk aversion under multiple sources of risk (Kihlstrom, Romer, and Williams, 1981; Pratt, 1988). Finally, technological relationships between expenditures on self-protection can be richer in a multiple risk setting than in environments with only one risk. For instance, when moving from two states of the world to four it is no longer obvious how to define a self-protective investment and our approach is but one suggestion. 


\section{References}

Briys, E., Y. Kahane, and Y. Kroll (1988): "Voluntary insurance coverage, compulsory insurance, and risky-riskless portfolio opportunities," Journal of Risk and Insurance, 55(4), 713-722.

Briys, E., H. Schlesinger, and M. G. V. D. Schulenburg (1991): "Reliability of risk management: Market structure, self-insurance and self-protection reconsidered," Geneva Papers on Risk and Insurance Theory, 16(1), 45-58.

Courbage, C., And B. Rey (2007): "Precautionary saving in the presence of other risks," Economic Theory, 32(2), 417-424.

(2012): "Optimal prevention and other risks in a two-period model," Mathematical Social Sciences, 63(3), 213-217.

Courbage, C., B. Rey, And N. Treich (2013): "Prevention and precaution," in Handbook of Insurance, ed. by G. Dionne, Huebner International Series on Risk, Insurance, and Economic Security, chap. 8. Springer.

Dachraoui, K., G. Dionne, L. Eeckhoudt, and P. Godfroid (2004): "Comparative mixed risk aversion: Definition and application to self-protection and willingness to pay," Journal of Risk and Uncertainty, 29(3), 261-276.

Dionne, G., And L. Eeckhoudt (1985): "Self-insurance, self-protection and increased risk aversion," Economics Letters, 17(1-2), 39-42.

Doherty, N. A., And H. Schlesinger (1983a): "The optimal deductible for an insurance policy when initial wealth is random," Journal of Business, 56(4), 555-565.

Doherty, N. A., And H. Schlesinger (1983b): "Optimal insurance in incomplete markets," Journal of Political Economy, 91(6), 1045-1054.

Eeckhoudt, L., R. Huang, and L. Tzeng (2012): "Precautionary effort: A new look," Journal of Risk and Insurance, 79(2), 585-590.

Ehrlich, I., AND G. S. BeCKer (1972): "Market insurance, self-insurance, and selfprotection," Journal of Political Economy, 80(4), 623-648.

Gollier, C. (2001): The Economics of Risk and Time. The MIT Press, Cambridge, MA.

Hofmann, A., and R. Peter (2015a): "Multivariate prevention decisions: Safe today or sorry tomorrow?," Economics Letters, 128(3), 51-53. 
(2015b): "Self-insurance, self-protection, and saving: On consumption smoothing and risk management," Journal of Risk and Insurance (forthcoming).

Kihlstrom, R. E., D. Romer, and S. Williams (1981): "Risk aversion with random initial wealth," Econometrica, 49(4), 911-920.

LEE, K. (2012): "Background risk and self-protection," Economics Letters, 114(3), 262264.

Menegatti, M. (2009): "Optimal prevention and prudence in a two-period model," Mathematical Social Sciences, 58(3), 393-397.

Menegatti, M. (2012): "New results on optimal prevention of risk averse agents," Economics Bulletin, 32(3), 2166-2173.

(2014): "Optimal choice on prevention and cure: A new economic analysis," The European Journal of Health Economics, 15(4), 363-372.

Menegatti, M., And F. ReBessi (2011): "On the substitution between saving and prevention," Mathematical Social Sciences, 62(3), 176-182.

Mossin, J. (1968): "Aspects of rational insurance purchasing," Journal of Political Economy, 76(4), 553-568.

Pratt, J. W. (1988): "Aversion to one risk in the presence of others," Journal of Risk and Uncertainty, 1(4), 395-413.

REY, B. (2003): "A note on optimal insurance in the presence of a nonpecuniary background risk," Theory and Decision, 54(1), 73-83.

SCHLESinger, H. (1992): "Risk management decisions when effectiveness is unreliable," in Workers' Compensation Insurance: Claim Costs, Prices, and Regulation, pp. 307328. Springer.

Schlesinger, H., And N. A. Doherty (1985): "Incomplete markets for insurance: An overview," Journal of Risk and Insurance, 52(3), 402-423.

Sмith, V. (1968): "Optimal Insurance Coverage," Journal of Political Economy, 68, 68-77.

Sun, Y., And E. Frees (2013): "Joint ratemaking of automobile and homeowner insurance," Working Paper (University of Wisconsin-Madison).

vd SchulenburG, J.-M. G. (1986): "Optimal insurance purchasing in the presence of compulsory insurance and uninsurable risks," Geneva Papers on Risk and Insurance, $11(38), 5-16$. 
WANG, J., AND J. LI (2015): "Precautionary effort: another trait for prudence," Journal of Risk and Insurance (forthcoming). 


\section{Appendix: The Second-Order Conditions}

We will prove here that the assumption of self-protection technology $x$ providing a return at least as large as self-protection technology $y$, non-increasing returns on self-protection together with the assumption of $-q^{\prime \prime} / q^{\prime}>-p^{\prime} / p$ are sufficient for the second-order conditions to hold. Consider the first-order conditions that characterize the optimal bundle of self-protection expenditures. Then,

$$
\begin{aligned}
& T_{x x}=u^{\prime \prime}-p^{\prime \prime}(1-k q) \alpha-k p^{\prime \prime} q \beta<0 \\
& T_{y y}=u^{\prime \prime}-q^{\prime \prime}(1-k p) \gamma-k p q^{\prime \prime} \delta<0
\end{aligned}
$$

and after some algebra

$$
\begin{aligned}
\operatorname{det} \operatorname{Hess} T= & T_{x x} T_{y y}-T_{x y}^{2} \\
= & -u^{\prime \prime}\left[(1-k p) q^{\prime \prime} \gamma+k p q^{\prime \prime} \delta+p^{\prime \prime}(1-k q) \alpha+k p^{\prime \prime} q \beta+2 k p^{\prime} q^{\prime}(\alpha-\beta)\right] \\
& +p^{\prime \prime}(1-k p) q^{\prime \prime}(1-k q) \alpha \gamma+k p p^{\prime \prime} q^{\prime \prime}(1-k q) \alpha \delta+k p^{\prime \prime}(1-k p) q q^{\prime \prime} \beta \gamma \\
& +k^{2} p p^{\prime \prime} q q^{\prime \prime} \beta \delta-k^{2}\left(p^{\prime} q^{\prime}\right)^{2}(\alpha-\beta)^{2} .
\end{aligned}
$$

We want to find conditions under which the determinant of the Hessian is positive at an interior solution to guarantee maximality. The expression in square brackets can be rearranged to obtain

$$
p^{\prime \prime} \alpha+q^{\prime \prime} \gamma+k(\alpha-\beta)\left[2 p^{\prime} q^{\prime}-p q^{\prime \prime}-p^{\prime \prime} q\right]
$$

This is positive as long as $2 p^{\prime} q^{\prime}-p q^{\prime \prime}-p^{\prime \prime} q$ is non-positive which is equivalent to

$$
\frac{p}{p^{\prime}} \cdot \frac{q^{\prime \prime}}{q^{\prime}}+\frac{p^{\prime \prime}}{p^{\prime}} \cdot \frac{q}{q^{\prime}} \geq 2
$$

Now $-q^{\prime \prime} / q^{\prime} \geq-p^{\prime} / p$ is equivalent to having the first expression larger than 1 and the assumption that $x$ provides a return on self-protection at least as large as $y$ and that returns on self-protection are non-increasing ensure that the second expression is larger than $1 .^{21}$

21 Terms of the form $-p^{\prime \prime} / p^{\prime}$ measure by how much prevention efficiency is reduced at the margin when investing in self-protection, see also Hofmann and Peter (2015a). A second-order Taylor approximation reveals that for $\Delta x$ small

$$
\frac{p(x)-p(x+\Delta x)}{p(x)} \approx \sigma(x) \Delta x\left(1-\frac{1}{2}\left(-\frac{p^{\prime \prime}}{p^{\prime}}\right) \Delta x\right),
$$

so that roughly speaking high $-p^{\prime \prime} / p^{\prime}$ is "bad" because the efficiency of prevention decreases quickly. In this sense, the condition $-q^{\prime \prime} / q^{\prime} \geq-p^{\prime} / p$ implies that technology $y$ is less efficient than technology $x$ also in a second-order sense. 
The remaining five terms of the determinant of the Hessian can be reorganized as follows:

$$
\begin{aligned}
& k^{2} p p^{\prime \prime} q q^{\prime \prime}(\alpha-\beta)^{2}-k p^{\prime \prime} q q^{\prime \prime} \gamma(\alpha-\beta)-k p p^{\prime \prime} q^{\prime \prime} \alpha(\gamma-\delta) \\
& +p^{\prime \prime} q^{\prime \prime} \alpha \gamma-k^{2}(\alpha-\beta)^{2}\left(p p^{\prime \prime} q q^{\prime \prime}-\left(p^{\prime} q^{\prime}\right)^{2}\right) .
\end{aligned}
$$

The first four of these are positive, the last one is negative. Combining the first and the last of these and factoring out yields

$$
k^{2}(\alpha-\beta)^{2}\left(p p^{\prime \prime} q q^{\prime \prime}-\left(p^{\prime} q^{\prime}\right)^{2}\right),
$$

which is non-negative as long as $p^{\prime \prime} / p^{\prime} \cdot q^{\prime \prime} / q^{\prime} \geq p^{\prime} / p \cdot q^{\prime} / q$; this is ensured by non-increasing returns on self-protection expenditures. This proves that the assumptions made to sign the comparative statics results in the paper are sufficient to guarantee that the secondorder conditions for the maximization over both levels of self-protection expenditures are satisfied and that the solutions characterized by the first-order conditions are actually describing utility-maximizing choices. 